\title{
Time evolution of the Rabi Hamiltonian from the unexcited vacuum
}

\author{
R F Bishop and C Emary \\ Department of Physics, University of Manchester Institute of Science and Technology (UMIST), \\ PO Box 88, Manchester M60 1QD, UK
}

Received 9 March 2001, in final form 1 June 2001

Published 6 July 2001

Online at stacks.iop.org/JPhysA/34/5635

\begin{abstract}
The Rabi Hamiltonian describes a single mode of electromagnetic radiation interacting with a two-level atom. Using the coupled cluster method, we investigate the time evolution of this system from an initially empty field mode and an unexcited atom. We give results for the atomic inversion and field occupation, and find that the virtual processes cause the field to be squeezed. No anti-bunching occurs.
\end{abstract}

PACS numbers: $0365 \mathrm{C}, 3170 \mathrm{H}, 3280,4250 \mathrm{D}$

\section{Introduction}

The Rabi Hamiltonian plays an important role in quantum optics. It describes a two-level atom interacting with a single mode of quantized electromagnetic radiation via a dipole interaction [1]. It also finds wider application, describing a spin interacting with phonons in NMR [2], for example. It is also related to the static Lee model in field theory [3].

The field mode is described by bosonic annihilation and creation operators, $b$ and $b^{\dagger}$ respectively, which obey the usual commutation relation,

$$
\left[b, b^{\dagger}\right]=1 \text {. }
$$

The two-level atom is described by the pseudo-spin operators

$$
\begin{array}{rlrl}
\sigma^{z} & =\left(\begin{array}{cc}
1 & 0 \\
0 & -1
\end{array}\right) & \sigma^{+}=\left(\begin{array}{ll}
0 & 2 \\
0 & 0
\end{array}\right) & \sigma^{-}=\left(\begin{array}{ll}
0 & 0 \\
2 & 0
\end{array}\right) \\
\sigma^{x}=\frac{1}{2}\left(\sigma^{+}+\sigma^{-}\right) & \sigma^{y}=\frac{i}{2}\left(\sigma^{-}-\sigma^{+}\right) .
\end{array}
$$

With these definitions the Rabi Hamiltonian is given by

$$
H=\frac{1}{2} \omega_{0} \sigma^{z}+\omega b^{\dagger} b+g\left(\sigma^{+}+\sigma^{-}\right)\left(b^{\dagger}+b\right) .
$$

There is a conserved parity $\Pi$ associated with the Hamiltonian,

$$
\Pi \equiv \exp (\mathrm{i} \pi N) \quad N \equiv b^{\dagger} b+\frac{1}{2}\left(\sigma^{z}+1\right) .
$$


There is no proof that this Hamiltonian is integrable, although suggestive evidence does exist [4]. Consequently, investigation of the Rabi system requires approximations to be made.

The most widely used approach is to make the rotating wave approximation (RWA). This was first applied by Jaynes and Cummings [5], yielding the Jaynes-Cummings (JC) model. Under the RWA, one neglects the 'virtual terms' $\sigma^{+} b^{\dagger}$ and $\sigma^{-} b$, also known as the counterrotating terms. This leads to the excitation number $N$ as well as parity $\Pi$ being conserved, and renders the model soluble by a series of $2 \times 2$ diagonalizations.

Despite its frequent use in quantum optics the actual validity of the RWA for specific applications is usually highly questionable. For example, we know [7] that the energy spectrum of the Rabi Hamiltonian can be approximated by its JC counterpart in the RWA only for sufficiently small values of the coupling strength $g$, and that the width of this range decreases as one proceeds higher up the spectrum. There is a further concern regarding the RWA. Ford and O'Connell [8] investigated the system of a charged harmonic oscillator interacting with a reservoir consisting of an infinite number of oscillators within the RWA. They showed that the spectrum has no lower bound for all models of physical interest. This constitutes a violation of the second law of thermodynamics, as we can take energy from the oscillator bath (modeling the environment) without producing an effect upon it; i.e., we can still remove an infinite amount of energy from it.

The energy level spectrum of the full Hamiltonian has been investigated by several authors. The simplest approach is to use the configuration-interaction (CI) method, equivalent to a large-scale diagonalization in a suitably defined finite subspace of the full Hilbert space. This method has been used both by Graham and Höhnerbach [6] and by Kuś [9] in investigating possible quantum chaos signatures of the model. Lo et al [10] have given an analysis of the validity of the CI method. Reik and others [12] have adapted Judd's method [11] for the JahnTeller system for use with the Rabi Hamiltonian. Here, the Hamiltonian is translated into the Bargmann representation [13] and solutions of the resulting differential equations are sought. Whereas Judd originally used a power series ansatz, Reik uses a Neumann series. For certain couplings, the Neumann series terminates, giving isolated, exact solutions known as Juddian solutions. Elsewhere the series gives a useful, convergent approximation. The Juddian points are valuable for comparison of approximate techniques. Variational results have also been provided by Bishop et al [14] and by Benivegna and Messina [15]. The latter method also permits perturbative corrections, allowing the exact results to be approached.

The time evolution of the Rabi Hamiltonian has been of considerable interest for a long time. We mention in particular the pioneering studies of Shirley [16]. Somewhat later, Eberly and co-workers [17] were the first to fully demonstrate the rich time evolution of this model. They worked within the RWA and discovered the collapse-revival nature of the subsequent evolution when the system is started from a coherent field [18]. The impact of the non-RWA terms on the time evolution has been investigated using various techniques. For example, Zaheer and Zubairy have used path-integral methods [19], while several authors have used perturbative techniques $[20,21]$. The drawback of these latter methods is their restriction to small coupling. Finally, Swain has given a formally exact result for the time evolution of the Rabi Hamiltonian [22]. However, the solutions are expressed as continued fractions, which limits their usefulness in practice.

In this paper we shall investigate the evolution of the Rabi system from an initial state composed of an empty field mode and an unexcited atom, which we denote as $|0, \downarrow\rangle$ and henceforth call the unexcited vacuum state. Within the RWA, the system would remain in this state indefinitely. However, inclusion of the counter-rotating terms means that we allow 'energy non-conserving' processes to occur. It is these virtual processes that drive the evolution of the system. For simplicity, we shall only consider the resonant case, $\omega=\omega_{0}$. 
In order to investigate the evolution of the Rabi system from the state $|0, \downarrow\rangle$ we employ here one of the most versatile and most accurate semi-analytical formalisms of microscopic quantum many-body theory, namely the coupled cluster method (CCM) [23-30]. Coupled cluster techniques are widely regarded as being amongst the most powerful of all $a b$ initio quantum many-body methods. As such they provide a number of distinct advantages over more traditional or more specialized methods which have hitherto been used in quantum optics and allied fields and, more specifically, to study Rabi systems. The CCM exists in two versions, the so-called normal (NCCM) and extended (ECCM) types [26], and in the present paper we employ only the former version.

A particular advantage of the CCM, a nonperturbative method originally developed in nuclear physics by Coester and Kümmel [23], is that it has been extremely widely applied and tested on a huge variety of physical systems [28]. These include areas as diverse as nuclear and subnuclear physics, quantum field theories (both in the spatial continuum and on the spatial lattice), condensed matter physics, quantum magnetism, and quantum chemistry. In almost all such cases CCM techniques now provide numerical results that are either the best or among the best available.

A pertinent, but quite typical, example is the electron gas, one of the most intensely studied of all quantum many-body problems. Here, the CCM results $[25,31,32]$ for the correlation energy, for example, agree over the entire metallic density range to within less than one millihartree per electron (or $<1 \%$ ) with the essentially exact Green function Monte Carlo results that are, very exceptionally, available for this fermionic system. The CCM results have never been bettered by any other technique. Elsewhere, in quantum chemistry, for example, after its early introduction by Čižek [33], the influence of the CCM has been profound, to the point where it is nowadays the method of choice for most highly accurate chemical studies [34,35].

Since the Rabi Hamiltonian involves a two-level system which is modelled by Pauli pseudospin operators, we note finally that the CCM has also been very successfully applied to a large number of spin-lattice systems exhibiting anti-ferromagnetic and other forms of magnetic ordering. In instances such as unfrustrated models on bipartite lattices where a Marshall-Peierls sign rule [36] exists (which provides a means to circumvent the infamous 'minus-sign problem' inherent in simulating many-fermion systems by quantum Monte Carlo (QMC) techniques), the CCM provided results fully comparable in accuracy to those obtained by QMC means [37]. The CCM is even able to predict with good accuracy the positions of the phase boundaries which mark the quantum phase transitions between states of different quantum order. Furthermore, the CCM provides equally accurate results for systems which are frustrated either geometrically (e.g. on a triangular lattice) [37], or dynamically (e.g. by competing interactions on different bonds on the lattice) [38], where QMC methods are much more difficult to apply. In several such instances the CCM results are now the best available.

Although the time-dependent formalism of the CCM [26] has existed for a considerable time, it has not yet found the same range of applications as its static counterpart. For example, Hoodbhoy and Negele [39] have investigated the application of the technique in nuclear dynamics, using two interacting Lipkin Hamiltonian systems as a test model. It has also found more application in chemical physics [40,41]. For example, Monkhorst has outlined the application of the time-dependent CCM to the treatment of molecular eigenstates [42], with the aim of describing such phenomena as scattering, chemical dynamics and laser chemistry. Sree Latha and Durga Prasad have investigated the application of the technique to multi-mode systems with vibronic coupling [43], a mechanism describing, amongst other things, nonadiabatic phenomena in the electronic spectroscopy of polyatomic molecules. We note that all of these studies have tended to only use the lowest levels of CCM approximation, due to the 
complexity of the systems studied. In comparison, we shall use high levels of approximation in an endeavour to describe the Rabi system as accurately as possible.

For other applications of the CCM the interested reader is referred to the reviews contained in $[28,30]$. An important feature of all the applications is that the results provided by the CCM are able to be systematically improved upon via well defined hierarchies of approximations. At each level of approximation the results are open to ready physical interpretation in terms of the correlated many-body clusters involved and their corresponding multiconfigurational creation operators. To date the NCCM has already been used to investigate the ground state of the Rabi Hamiltonian [44], where it has been shown to give excellent results for values of the coupling parameter $g \lesssim 0.665$. In view of the demonstrable success in treating so many other many-body systems, we now wish to apply it to the dynamical evolution of Rabi systems, with a particular aim to shed light on the importance of the counter-rotating terms dropped in the commonly made RWA.

\section{Formalism}

We now briefly describe the application of the normal CCM to the Rabi Hamiltonian of interest to us here.

Let $|\Psi(t)\rangle$ and $\langle\tilde{\Psi}(t)|$ be the exact ket and bra states at time $t$ for an arbitrary many-body quantum mechanical system, chosen so that

$$
\langle\tilde{\Psi}(t) \mid \Psi(t)\rangle=1 \quad \forall t .
$$

The Hilbert space for our system may be described in terms of a model state or cyclic vector $\left|\Phi_{0}\right\rangle$ and a corresponding complete set of mutually commuting multiconfigurational creation operators $\left\{C_{I}^{\dagger}\right\}$. The set $\left\{C_{I}^{\dagger}\right\}$ is defined with respect to the reference state, such that $C_{I}\left|\Phi_{0}\right\rangle=0=\left\langle\Phi_{0}\right| C_{I}^{\dagger}, \quad \forall I \neq 0$, in a notation in which $C_{0}^{\dagger} \equiv 1$, the identity operator. In general, $I$ is a set index and the operators $C_{I}^{\dagger}$ contain products of single-particle operators. The set index $\{I\}$ is complete in the sense that the set of states $\left\{C_{I}^{\dagger}\left|\Phi_{0}\right\rangle\right\}$ provides a complete basis for the Hilbert space. The reference state, $\left|\Phi_{0}\right\rangle$, must be chosen to be non-orthogonal to the actual wavefunction of the system,

$$
\left\langle\Phi_{0} \mid \Psi(t)\right\rangle \neq 0 \quad \forall t .
$$

Usually one chooses $\left\langle\Phi_{0}\left|C_{I} C_{J}^{\dagger}\right| \Phi_{0}\right\rangle=\delta_{I J}$, where $\delta_{I J}$ is some suitably defined Kronecker symbol. However, our later choice of $\left\{C_{I}^{\dagger}\right\}$ will leave the set $\left\{C_{I}^{\dagger}\left|\Phi_{0}\right\rangle\right\}$ orthogonal but not normalized;

$$
\left\langle\Phi_{0}\left|C_{I} C_{J}^{\dagger}\right| \Phi_{0}\right\rangle=N_{I} \delta_{I J}
$$

The ket and bra states are formally parametrized independently in the normal CCM as

$$
|\Psi(t)\rangle=\mathrm{e}^{k(t)} \mathrm{e}^{\hat{S}(t)}\left|\Phi_{0}\right\rangle \quad\langle\tilde{\Psi}(t)|=\mathrm{e}^{-k(t)}\left\langle\Phi_{0}\right| \hat{\tilde{S}} \mathrm{e}^{-\hat{S}}
$$

where

$$
\hat{S}=\sum_{I \neq 0} s_{I}(t) C_{I}^{\dagger} \quad \hat{\tilde{S}}=1+\sum_{I \neq 0} \tilde{s}_{I}(t) C_{I}
$$

and $k(t)$ is a $c$-number.

Using this parametrization, the expectation value of an arbitrary operator, $\hat{X}$, is given by

$$
\langle\hat{X}\rangle=\langle\tilde{\Psi}(t)|\hat{X}| \Psi(t)\rangle=\left\langle\Phi_{0}\left|\hat{\tilde{S}} \mathrm{e}^{-\hat{S}} \hat{X} \mathrm{e}^{\hat{S}}\right| \Phi_{0}\right\rangle
$$


In the evaluation of $\langle\hat{X}\rangle$, we use the nested commutator relation,

$$
\tilde{\hat{X}} \equiv \mathrm{e}^{-\hat{S}} \hat{X} \mathrm{e}^{\hat{S}}=\hat{X}+[\hat{X}, \hat{S}]+\frac{1}{2 !}[[\hat{X}, \hat{S}] \hat{S}]+\cdots
$$

This equation describes a similarity transformation of the operator $\hat{X}$. The similarity transformed Hamiltonian, $\tilde{H} \equiv \mathrm{e}^{-\hat{S}} \mathrm{He}^{\hat{S}}$, lies at the heart of the CCM. The ability of the NCCM to describe the Rabi stationary ground state has been investigated elsewhere $[44,45]$. Here we shall only consider the time-dependent formalism. To this end, we introduce the action functional

$$
A \equiv \int_{t_{0}}^{t_{1}}\left\{\left\langle\tilde{\Psi}(t)\left|\left(\mathrm{i} \frac{\partial}{\partial t}-H\right)\right| \Psi(t)\right\rangle\right\} .
$$

The stationarity principle,

$$
\frac{\delta A}{\delta|\Psi\rangle}=0=\frac{\delta A}{\delta\langle\tilde{\Psi}|}
$$

for all independent variations in the bra and ket states such that $\delta\left|\Psi\left(t_{j}\right)\right\rangle=0=\delta\left\langle\tilde{\Psi}\left(t_{j}\right)\right| ; j=$ 0,1 , reproduces the time-dependent Schrödinger equations,

$$
H|\Psi\rangle=\mathrm{i} \frac{\partial}{\partial t}|\Psi\rangle \quad\langle\tilde{\Psi}| H=-\mathrm{i} \frac{\partial}{\partial t}\langle\tilde{\Psi}| .
$$

Equation (14) gives Hamilton's equations of motion for the cluster coefficients,

$$
\mathrm{i} \frac{\mathrm{d} s_{I}}{\mathrm{~d} t}=\frac{1}{N_{I}} \frac{\partial\langle H\rangle}{\partial \tilde{s}_{I}} \quad-\mathrm{i} \frac{\mathrm{d} \tilde{s}_{I}}{\mathrm{~d} t}=\frac{1}{N_{I}} \frac{\partial\langle H\rangle}{\partial s_{I}}
$$

where $N_{I}$ is the norm from equation (8).

Following [44] we make the following choice of reference state and operators:

$$
\left|\Phi_{0}\right\rangle=|0, \downarrow\rangle \quad \hat{S}=\hat{S}_{1}+\hat{S}_{2}
$$

with

$$
\hat{S}_{1}=\sum_{n=1}^{\infty} s_{n}^{(1)}(t)\left(b^{\dagger}\right)^{n} \quad \hat{S}_{2}=\sum_{n=1}^{\infty} s_{n}^{(2)}(t)\left(b^{\dagger}\right)^{n-1} \sigma^{+} .
$$

The corresponding expansion for $\hat{\tilde{S}}$ is

$$
\hat{\tilde{S}}=1+\hat{\tilde{S}}_{1}+\hat{\tilde{S}}_{2}
$$

with

$$
\hat{\tilde{S}}_{1}=\sum_{n=1}^{\infty} \tilde{s}_{n}^{(1)}(t) b^{n} \quad \hat{\tilde{S}}_{2}=\sum_{n=1}^{\infty} \tilde{s}_{n}^{(2)}(t) b^{n-1} \sigma^{-} .
$$

We note that the states $\left(b^{\dagger}\right)^{n}|0, \downarrow\rangle$ and $\left(b^{\dagger}\right)^{(n-1)} \sigma^{+}|0, \downarrow\rangle$ are eigenstates of the excitation operator, $N$, with eigenvalues equal to $n$. They have corresponding even or odd parity (i.e. eigenvalues of $\Pi$ equal to +1 or -1 , respectively) depending on whether $n$ is even or odd. The reference state $\left|\Phi_{0}\right\rangle$ has positive parity. This is the same as the ground state, as demanded by equation (7). The overlaps, $N_{I}$, of the operators (18) are

$$
\begin{aligned}
& N_{n}^{(1)}=\left\langle 0, \downarrow\left|b^{n}\left(b^{\dagger}\right)^{n}\right| 0, \downarrow\right\rangle=n ! \\
& N_{n}^{(2)}=\left\langle 0, \downarrow\left|\sigma^{-} b^{n-1}\left(b^{\dagger}\right)^{n-1} \sigma^{+}\right| 0, \downarrow\right\rangle=4(n-1) ! .
\end{aligned}
$$

This choice of reference state and cluster operators has been found to predict a spurious phase transition at $g \rightarrow g_{\mathrm{c}} \approx 0.665$ [10]. This in fact signals a breakdown of the calculation for $g>g_{\mathrm{c}}$. For $g<g_{\mathrm{c}}$ this scheme gives excellent results for the ground state, and so it is within this region that we shall work. 
Table 1. Convergence of the method with decreasing step size. The values of the real and imaginary parts of two typical coefficients are shown at time $g t=1$, for a SUB-12 calculation with $g=0.05$.

\begin{tabular}{lllll}
\hline Step size & $\mathbb{R}\left\{s_{2}^{(2)}\right\}$ & $\mathbb{I}\left\{s_{2}^{(2)}\right\}$ & $\mathbb{R}\left\{s_{8}^{(2)}\right\}$ & $\mathbb{I}\left\{s_{8}^{(2)}\right\}$ \\
\hline 0.0333 & $-9.168663 \times 10^{-3}$ & $-1.772499 \times 10^{-2}$ & $2.946230 \times 10^{-8}$ & $-2.517358 \times 10^{-8}$ \\
0.0250 & $-9.167811 \times 10^{-3}$ & $-1.772437 \times 10^{-2}$ & $2.365733 \times 10^{-8}$ & $-2.493248 \times 10^{-8}$ \\
0.0200 & $-9.167578 \times 10^{-3}$ & $-1.772420 \times 10^{-2}$ & $2.212346 \times 10^{-8}$ & $-2.482074 \times 10^{-8}$ \\
0.0100 & $-9.167426 \times 10^{-3}$ & $-1.772409 \times 10^{-2}$ & $2.114473 \times 10^{-8}$ & $-2.473925 \times 10^{-8}$ \\
0.0050 & $-9.167416 \times 10^{-3}$ & $-1.772408 \times 10^{-2}$ & $2.108448 \times 10^{-8}$ & $-2.473400 \times 10^{-8}$ \\
0.0033 & $-9.167416 \times 10^{-3}$ & $-1.772408 \times 10^{-2}$ & $2.108127 \times 10^{-8}$ & $-2.473372 \times 10^{-8}$ \\
0.0025 & $-9.167416 \times 10^{-3}$ & $-1.772408 \times 10^{-2}$ & $2.108073 \times 10^{-8}$ & $-2.473367 \times 10^{-8}$ \\
0.0010 & $-9.167416 \times 10^{-3}$ & $-1.772408 \times 10^{-2}$ & $2.108048 \times 10^{-8}$ & $-2.473365 \times 10^{-8}$ \\
0.0005 & $-9.167416 \times 10^{-3}$ & $-1.772408 \times 10^{-2}$ & $2.108048 \times 10^{-8}$ & $-2.473365 \times 10^{-8}$ \\
\hline
\end{tabular}

Table 2. Convergence of the SUB- $N$ method with increasing truncation index $N$. The values of the real and imaginary parts of two typical coefficients are shown at time $g t=1$, for $g=0.05$. The step size was 0.0005 . This convergence is only valid for small couplings.

\begin{tabular}{rllll}
\hline$N$ & $\mathbb{R}\left\{s_{2}^{(2)}\right\}$ & $\mathbb{I}\left\{s_{2}^{(2)}\right\}$ & $\mathbb{R}\left\{s_{8}^{(2)}\right\}$ & $\mathbb{I}\left\{s_{8}^{(2)}\right\}$ \\
\hline 2 & $-9.050635 \times 10^{-3}$ & $-1.783754 \times 10^{-2}$ & - & - \\
4 & $-9.167532 \times 10^{-3}$ & $-1.772413 \times 10^{-2}$ & - & - \\
6 & $-9.167416 \times 10^{-3}$ & $-1.772408 \times 10^{-2}$ & - & - \\
8 & $-9.167416 \times 10^{-3}$ & $-1.772408 \times 10^{-2}$ & $1.675505 \times 10^{-8}$ & $4.392506 \times 10^{-9}$ \\
10 & $-9.167416 \times 10^{-3}$ & $-1.772408 \times 10^{-2}$ & $2.126271 \times 10^{-8}$ & $-2.456155 \times 10^{-8}$ \\
12 & $-9.167416 \times 10^{-3}$ & $-1.772408 \times 10^{-2}$ & $2.108048 \times 10^{-8}$ & $-2.473365 \times 10^{-8}$ \\
14 & $-9.167416 \times 10^{-3}$ & $-1.772408 \times 10^{-2}$ & $2.107987 \times 10^{-8}$ & $-2.473357 \times 10^{-8}$ \\
16 & $-9.167416 \times 10^{-3}$ & $-1.772408 \times 10^{-2}$ & $2.107987 \times 10^{-8}$ & $-2.473356 \times 10^{-8}$ \\
18 & $-9.167416 \times 10^{-3}$ & $-1.772408 \times 10^{-2}$ & $2.107987 \times 10^{-8}$ & $-2.473356 \times 10^{-8}$ \\
20 & $-9.167416 \times 10^{-3}$ & $-1.772408 \times 10^{-2}$ & $2.107987 \times 10^{-8}$ & $-2.473356 \times 10^{-8}$ \\
40 & $-9.167416 \times 10^{-3}$ & $-1.772408 \times 10^{-2}$ & $2.107987 \times 10^{-8}$ & $-2.473356 \times 10^{-8}$ \\
\hline
\end{tabular}

\section{Time evolution}

We shall start the system in the unexcited vacuum, $|0, \downarrow\rangle$. This corresponds to the initial, $t=t_{0}$, conditions

$$
s_{n}^{(1)}\left(t_{0}\right)=s_{n}^{(2)}\left(t_{0}\right)=0=\tilde{s}_{n}^{(1)}\left(t_{0}\right)=\tilde{s}_{n}^{(2)}\left(t_{0}\right) .
$$

Other choices are certainly possible, but the above choice is the most obvious one that corresponds to a physical state and which satisfies equation (7).

In the evaluation of the time evolution equations we truncate the sums in equations (18) and (20) at $n=N$, giving the so-called SUB- $N$ approximation, in which all the coefficients $s_{n}^{(i)}(t)$ and $\tilde{s}_{n}^{(i)}(t), i=1,2$, for $n>N$ are set to zero. The resulting set of coupled equations (16) is then solved numerically by taking finite time steps. We note that since the initial state $|0, \downarrow\rangle$ has even parity $\Pi$, all the coefficients $s_{n}^{(i)}(t)$ and $\tilde{s}_{n}^{(i)}(t), i=1,2$, vanish identically for odd values of $n$, at all times. The convergence of our method with decreasing step size is demonstrated in table 1 . Naively, one would expect a similar type of convergence with increasing SUB- $N$ level. Adding more cluster operators should allow the actual wavefunction to be described more accurately. However, we find that this is only the case for small coupling, as shown in table 2. In general we find that for a given coupling parameter, $g$, there is a maximal value of the truncation index $N$ for which convergence occurs. For higher values of $N$ the method diverges, with the calculated cluster coefficients diverging to infinity. To understand 


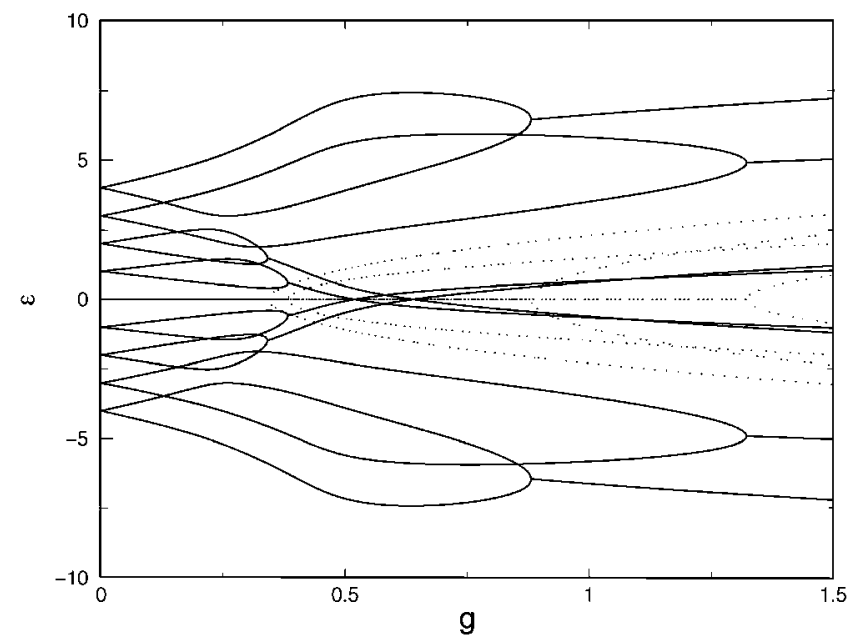

Figure 1. The excitation-energy spectrum of the resonant Rabi Hamiltonian $\left(\omega=\omega_{0}=1\right)$ as a function of $g$, as determined by a SUB-4 NCCM calculation. Solid (dotted) curves show real (imaginary) parts of the excitation energies. The negative-energy solutions and those with imaginary components are spurious.

this behaviour we look at the energy spectrum calculated by the NCCM under this scheme. This spectrum is determined by first calculating the ground-state coefficients, $\left\{s_{I}, \tilde{s_{I}}\right\}$, and using these to construct the so-called dynamic matrix, $H_{\mathrm{D}}$ of linear response theory [30]. This matrix is then diagonalized to give the energy eigenvalues. A full discussion of this procedure would take us too far afield and so we shall just consider the results and refer the reader to [30] for further information. Figure 1 shows the excitation energies as determined by a SUB-4 calculation. A SUB- $N$ calculation yields $4 N$ eigenvalues, half of which are spurious negative energy solutions, generated as a result of the symmetry of $H_{\mathrm{D}}$.

The important feature to note is that at certain values of $g$ (e.g. at $g \approx 0.35$ for the SUB-4 case in figure 1), which depend on the truncation index $N$, two real energy levels come together, and as $g$ increases, become a complex conjugate pair. Thus, for higher values of $g$, the NCCM predicts a pair of complex conjugate energy eigenvalues for the Hamiltonian. We can see what this means for the time evolution by considering the basic quantum mechanical expression for the evolution of an arbitrary wavefunction,

$$
|\Psi(t)\rangle=\sum_{n}\left\langle u_{n} \mid \Psi\left(t_{0}\right)\right\rangle \mathrm{e}^{-\mathrm{i} E_{n}\left(t-t_{0}\right)}\left|u_{n}\right\rangle
$$

where $\left|u_{n}\right\rangle$ is the eigen-ket corresponding to energy eigenvalue $E_{n}$. We see that having imaginary components to $E_{n}$ of both signs will lead to exponentially growing terms in this sum, as opposed to bounded oscillations. Thus we expect our NCCM time evolution calculations to break down for a given coupling if the SUB- $N$ level predicts complex energy eigenvalues at that coupling. Figure 2 shows the values of $g$, for a given SUB- $N$ truncation index $N$, above which the spectrum contains imaginary components. These are the points where our time-evolution calculations break down. The SUB-2 time evolution breaks down at exactly the point where the ground-state calculation does.

Sree Latha and Durga Prasad have encountered similar problems in their applications of the closely related multi-reference time-dependent coupled cluster method (MRTDCCM) [46], which also posits an exponential ansatz for the wavefunction, but uses a model space spanned by a number of states as opposed to the NCCMs single reference state. They trace the origin 


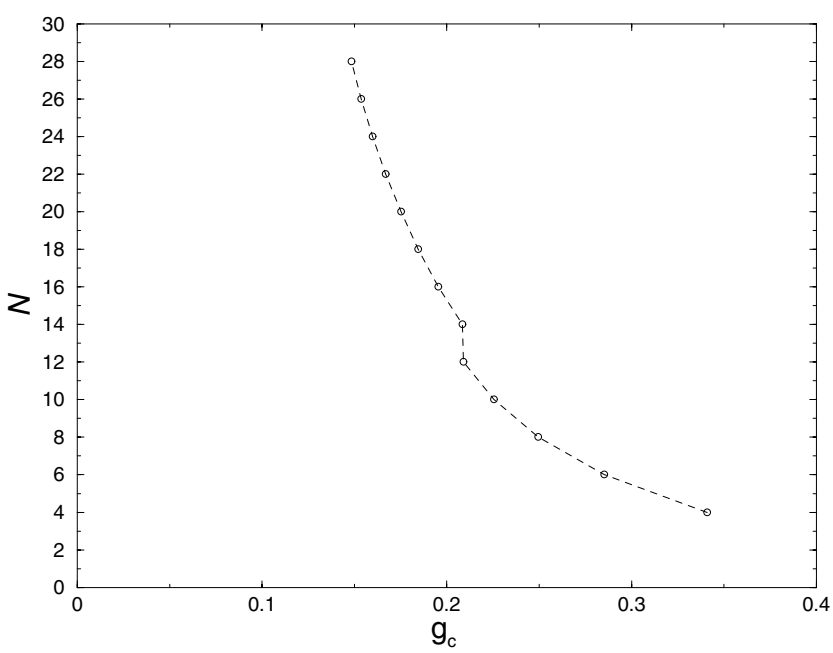

Figure 2. The critical value $g_{\mathrm{c}}=g_{\mathrm{c}}(N)$ of the coupling parameter, above which the NCCM timeevolution calculations break down, is shown for various values of the SUB- $N$ truncation index $N$. This is the coupling above which the SUB- $N$ excitation spectrum develops imaginary components. The Hamiltonian is resonant $\left(\omega=\omega_{0}=1\right)$.

of the complex eigenvalues back to the use of the similarity-transformed Hamiltonian $\tilde{H}$, which, when truncated, is not necessarily Hermitian. This lack of Hermiticity permits complex eigenvalues, whose corresponding eigenvectors are related to the the so-called 'intruder states'. They conjecture that this situation may be expected to arise when the reference state or space interacts strongly with a state or set of states in the rest of the Hilbert space; which is just the situation we observe here.

In understanding the following results, it will be useful to study a Fourier transforms of the time series. Terms similar to

$$
f(t)=\sum_{k=1}^{N}(k-1) ! s_{k}^{(2)}(t) \tilde{s}_{k}^{(2)}(t)
$$

occur in all the quantities that we look at below. We define $F(\Omega)$ as the Fourier transform of $f(t)$ and figure 3 shows a plot of this quantity for typical parameters. We see a discrete spectrum with three main peaks. These peaks correspond to the three lowest positive-parity energy levels in the spectrum, as one would expect. This means that the time evolution will be quasi-periodic. This quasi-periodic structure is reflected in the behaviour of the cluster coefficients, as can be clearly seen from the typical parametric plot shown in figure 4.

\section{Atomic inversion}

The atomic inversion, $\left\langle\sigma^{z}\right\rangle$, has been the primary atomic quantity of interest when studying the Rabi system, not least because it is experimentally determinable [47]. In our NCCM scheme the atomic inversion is given by

$$
\left\langle\sigma^{z}\right\rangle=-1+8 \sum_{n=1}^{\infty}(n-1) ! s_{n}^{(2)} \tilde{s}_{n}^{(2)} .
$$

Being an observable, $\left\langle\sigma^{z}\right\rangle$ should always be real but the cluster-coefficients $s_{n}^{(i)}$ and $\tilde{s}_{n}^{(i)}$, $i=1,2$, are, in general, complex. The truncation of the cluster operators leads to the exact 


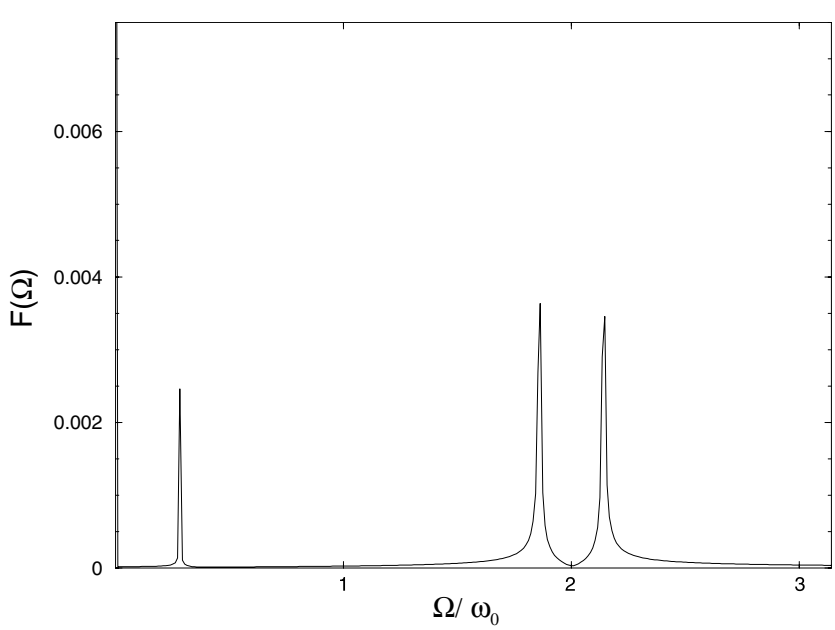

Figure 3. Fourier transform of the quantity $f(t)$ from equation (24) for the resonant Hamiltonian $\left(\omega=\omega_{0}=1\right)$ with $g=0.05$. This shows a discrete spectrum with three main frequency components.

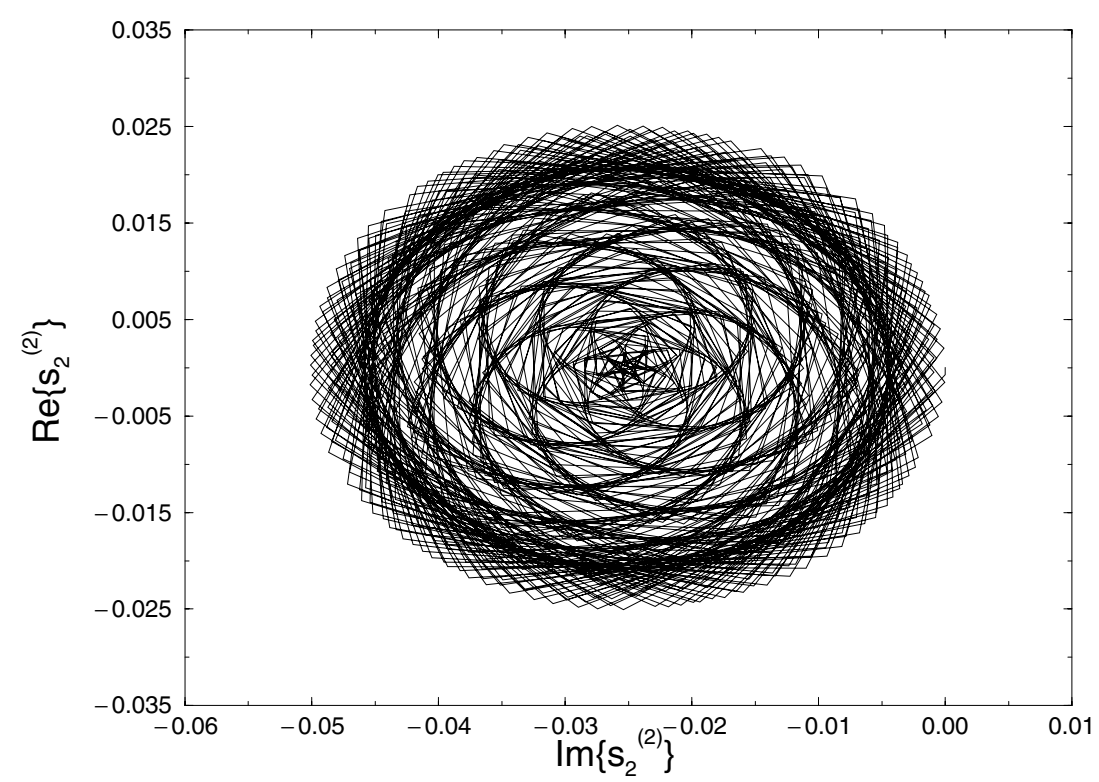

Figure 4. A parametric 'phase-space' plot of the coefficient $s_{2}^{(2)}(t)$, shown for a range of the time parameter $t$ for which $0 \leqslant g t \leqslant 120\left(g=0.05\right.$, SUB-30, $\left.\omega=\omega_{0}=1\right)$.

Hermiticity of the bra and ket states being broken. This in turn means that the atomic inversion calculated under the NCCM is not constrained to be real. Calculations show that $s_{2}^{(2)} \tilde{s}_{2}^{(2)}$ is the dominant contribution to the sum in equation (25) and reveal these two coefficients to be almost complex conjugate to one other. Subsequent terms in the summation conspire to reduce the size of the spurious imaginary part, this reduction becoming more perfect with increasing truncation. For small couplings we can almost completely eliminate this imaginary part, although the restriction on the maximum SUB- $N$ level permissible for higher couplings 

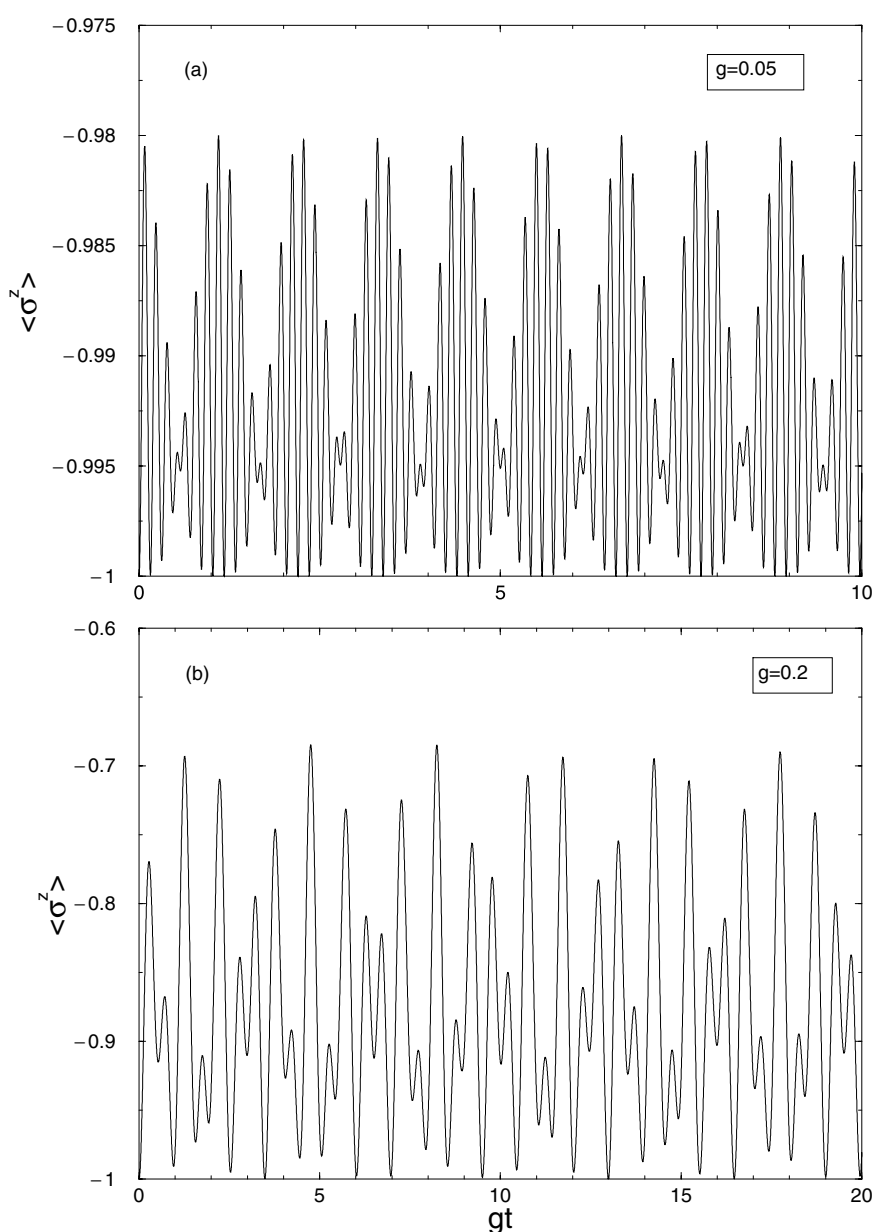

Figure 5. Time evolution of the atomic inversion, $\left\langle\sigma^{z}\right\rangle$, for two different couplings: $(a) g=0.05$, SUB-30 and $(b) g=0.2$, SUB-14. The Hamiltonian is resonant $\left(\omega=\omega_{0}=1\right)$.

means that we cannot eliminate this component entirely as $g$ becomes larger. The complex part of the calculated $\left\langle\sigma^{z}\right\rangle$ is small and can be used as a rough measure of the error in the NCCM calculations. For the parameters used here, $\operatorname{RMS}\left(\mathbb{I}\left\{\left\langle\sigma^{z}\right\rangle\right\}\right) \approx 2 \times 10^{-13}, \operatorname{Max}\left(\mathbb{I}\left\{\left\langle\sigma^{z}\right\rangle\right\}\right) \approx$ $2 \times 10^{-11}$ for $g=0.05$ and $\operatorname{RMS}\left(\mathbb{I}\left\{\left\langle\sigma^{z}\right\rangle\right\}\right) \approx 1 \times 10^{-6}, \operatorname{Max}\left(\mathbb{I}\left\{\left\langle\sigma^{z}\right\rangle\right\}\right) \approx 4 \times 10^{-4}$ for $g=0.2$. Figure 5 shows the evolution of the atomic inversion for two different couplings, $g=0.05$ and 0.2 .

\section{Field observables}

\subsection{Photon number}

The most important operator associated with the field is $b^{\dagger} b$, the photon number operator. In terms of the NCCM coefficients, it has an expectation value $\bar{n}(t)$,

$$
\bar{n}(t)=\left\langle b^{\dagger} b\right\rangle=\sum_{n=1}^{\infty} n n ! s_{n}^{(1)} \tilde{s}_{n}^{(1)}+4 \sum_{n=1}^{\infty}(n-1)(n-1) ! s_{n}^{(2)} \tilde{s}_{n}^{(2)} .
$$



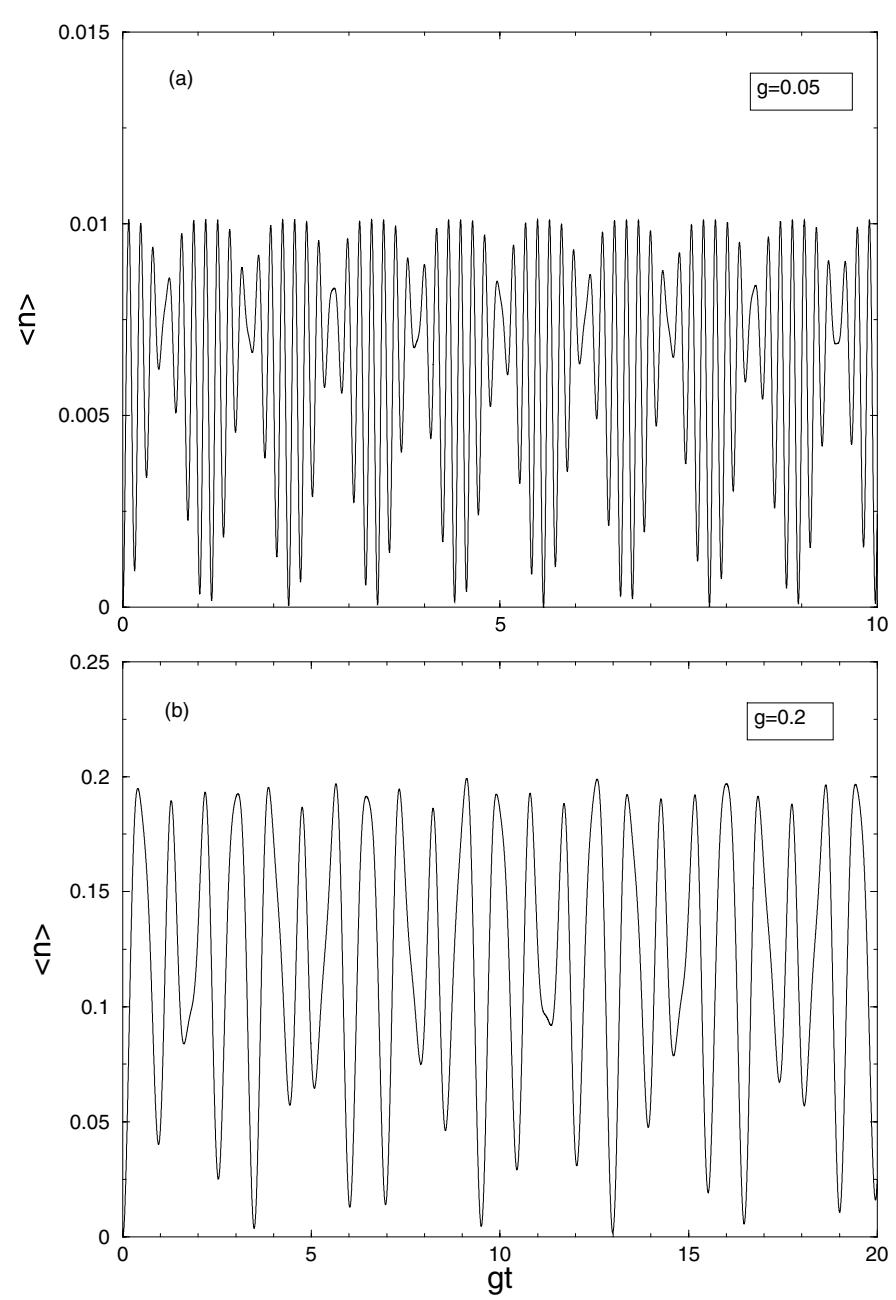

Figure 6. Time evolution of the field occupation number $\langle n\rangle \equiv\left\langle b^{\dagger} b\right\rangle$, using the same parameters as in figure 5. The Hamiltonian is resonant $\left(\omega=\omega_{0}=1\right)$.

Figure 6 shows the evolution of this quantity. The nature of this evolution is very similar to that of the atomic inversion, and we clearly observe the atom exchanging energy with the field. A time average of $\left\langle b^{\dagger} b\right\rangle$ provides an estimate of the mean number of virtual photons in the cavity at any given moment. For example, for $g=0.05, \overline{\left\langle b^{\dagger} b\right\rangle} \approx 0.0063$ and for $g=0.2$, $\overline{\left\langle b^{\dagger} b\right\rangle} \approx 0.12$.

\subsection{Photon anti-bunching}

The next field observable we shall study is the second-order correlation function,

$$
g^{(2)}(\tau)=\frac{\left\langle b^{\dagger}(t) b^{\dagger}(t+\tau) b(t+\tau) b(t)\right\rangle}{\left\langle b^{\dagger}(t) b(t)\right\rangle^{2}}
$$


This allows us to study whether the field exhibits anti-bunching. For this purpose, we only require $g^{(2)}(0)$,

$$
g^{(2)}(0)=\frac{\left\langle b^{\dagger} b^{\dagger} b b\right\rangle}{\left\langle b^{\dagger} b\right\rangle^{2}}
$$

and we define the convenient parameter $[48,49]$

$$
y \equiv\left\langle b^{\dagger} b^{\dagger} b b\right\rangle-\left\langle b^{\dagger} b\right\rangle^{2} .
$$

A value of $y<0$ corresponds to $g^{(2)}(0)<1$ and indicates an anti-bunched field.

For the NCCM, $\left\langle b^{\dagger} b^{\dagger} b b\right\rangle$ is given by

$$
\begin{aligned}
\left\langle b^{\dagger} b^{\dagger} b b\right\rangle=\sum_{n=1}^{\infty} n(n-1) n ! s_{n}^{(1)} \tilde{s}_{n}^{(1)}+\sum_{n, m=1}^{\infty} n m(n+m) ! s_{n}^{(1)} s_{m}^{(1)} \tilde{s}_{n+m}^{(1)} \\
+4 \sum_{n=1}^{\infty}(n-1)(n-2)(n-1) ! s_{n}^{(2)} \tilde{s}_{n}^{(2)} \\
+8 \sum_{n, m=1}^{\infty} n(m-1)(n+m-1) ! s_{n}^{(1)} s_{m}^{(2)} \tilde{s}_{n+m}^{(2)} .
\end{aligned}
$$

Figure 7 shows the quantity $y$ for various couplings. For small values of $g$ we see that $y$ never drops below zero and thus the field is never anti-bunched. For higher values of coupling, we see that $y$ occasionally does fall very slightly below zero. This effect is extremely small and we believe that it is due to inaccuracies introduced by using small truncation levels.

\subsection{Squeezing}

By analogy to the position and momentum operators of the harmonic oscillator, we introduce the following quadrature operators for the electromagnetic field [50]:

$$
\begin{aligned}
Q_{1} & \equiv \frac{1}{2}\left[b \exp (\mathrm{i} \omega t)+b^{\dagger} \exp (-\mathrm{i} \omega t)\right] \\
Q_{2} & \equiv \frac{1}{2 \mathrm{i}}\left[b \exp (\mathrm{i} \omega t)-b^{\dagger} \exp (-\mathrm{i} \omega t)\right] .
\end{aligned}
$$

Their variances, given by

$$
\begin{aligned}
& \left(\Delta Q_{1}\right)^{2}=\frac{1}{2}\left\{\left\langle b^{\dagger} b\right\rangle+\mathbb{R}\left[\left\langle b^{2}\right\rangle \exp (2 \mathrm{i} \omega t)\right]\right\}-\{\mathbb{R}[\langle b\rangle \exp (\mathrm{i} \omega t)]\}^{2}+\frac{1}{4} \\
& \left(\Delta Q_{2}\right)^{2}=\frac{1}{2}\left\{\left\langle b^{\dagger} b\right\rangle-\mathbb{R}\left[\left\langle b^{2}\right\rangle \exp (2 \mathrm{i} \omega t)\right]\right\}-\{\mathbb{I}[\langle b\rangle \exp (\mathrm{i} \omega t)]\}^{2}+\frac{1}{4}
\end{aligned}
$$

satisfy the uncertainty relation,

$$
\Delta Q_{1} \Delta Q_{2} \geqslant \frac{1}{4} \text {. }
$$

The field is said to be squeezed when either $\left(\Delta Q_{1}\right)^{2}$ or $\left(\Delta Q_{2}\right)^{2}<\frac{1}{4}$. The NCCM expressions for the remaining expectation values in equation (32) are easily calculated, and are given by

$$
\begin{aligned}
&\left\langle b^{2}\right\rangle=2 s_{2}^{(1)}+ s_{1}^{(1)} s_{1}^{(1)}+\sum_{n=3}^{\infty} n ! s_{n}^{(1)} \tilde{s}_{n-2}^{(1)} \\
&+\sum_{n=1}^{\infty} \sum_{m=1}^{\infty} n m(n+m-2) ! s_{n}^{(1)} s_{m}^{(1)} \tilde{s}_{n+m-2}^{(1)}\left(1-s_{n 1} s_{m 1}\right) \\
&+4 \sum_{n=3}^{\infty}(n-1) ! s_{n}^{(2)} \tilde{s}_{n-2}^{(2)}+8 \sum_{n=1}^{\infty} \sum_{m=2}^{\infty} n(m-1)(n+m-3) ! s_{n}^{(1)} s_{m}^{(2)} \tilde{s}_{n+m-2}^{(2)} \\
&\langle b\rangle=s_{1}^{(1)}+\sum_{n=2}^{\infty} n ! s_{n}^{(1)} \tilde{s}_{n-1}^{(1)}+4 \sum_{n=2}^{\infty}(n-1) ! s_{n}^{(2)} \tilde{s}_{n-1}^{(2)} .
\end{aligned}
$$



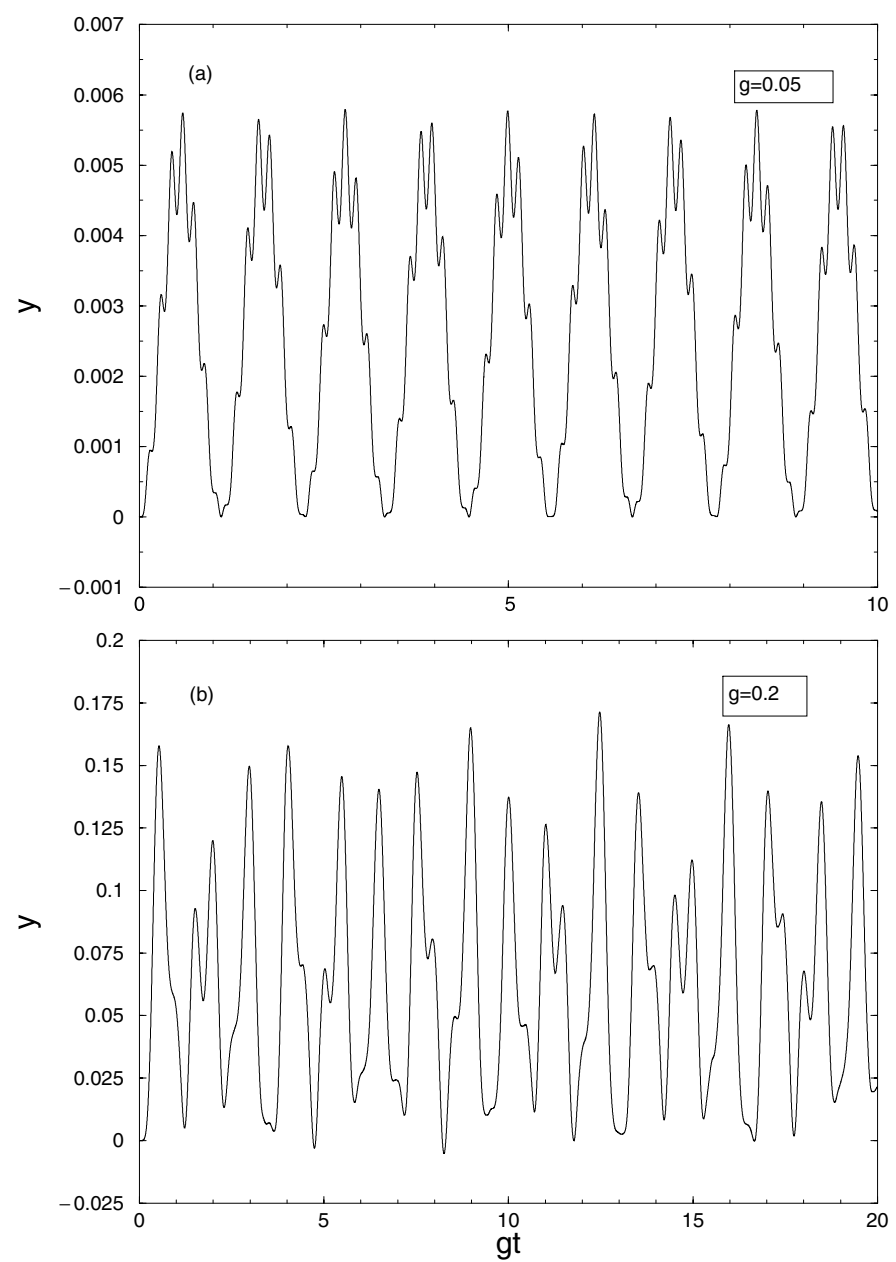

Figure 7. Time evolution of the photon anti-bunching measure, $y$, using the same parameters as figure 5. Note that $y<0$ indicates anti-bunching. The Hamiltonian is resonant $\left(\omega=\omega_{0}=1\right)$.

Figure 8 shows the evolution of these variances from the vacuum. For $g=0.05$, we see that the values of $\left(\Delta Q_{i}\right)^{2} ; i=1,2$, only just drop below $\frac{1}{4}\left(\min \left(\Delta Q_{i}\right)^{2}=0.2495\right.$ for $g=0.05)$. However, for greater couplings, we do see that squeezing is more significant $\left(\min \left(\Delta Q_{i}\right)^{2}=0.1351\right.$ for $\left.g=0.2\right)$.

\section{Discussion}

We have demonstrated the ability of the NCCM to describe the time evolution of a simple but important quantum system. We have also outlined the limitations of the method. Despite these, we have been able to obtain a range of useful results for the system.

We have seen that the counter-rotating terms give rise to quite complex behaviour in the evolution from the unexcited vacuum. The field, although not anti-bunched, does exhibit squeezing, which becomes more pronounced with increased coupling. However, the small absolute magnitude of these effects and the limitations of the model, such as neglect of thermal photons, clouds the experimental significance of the results. 

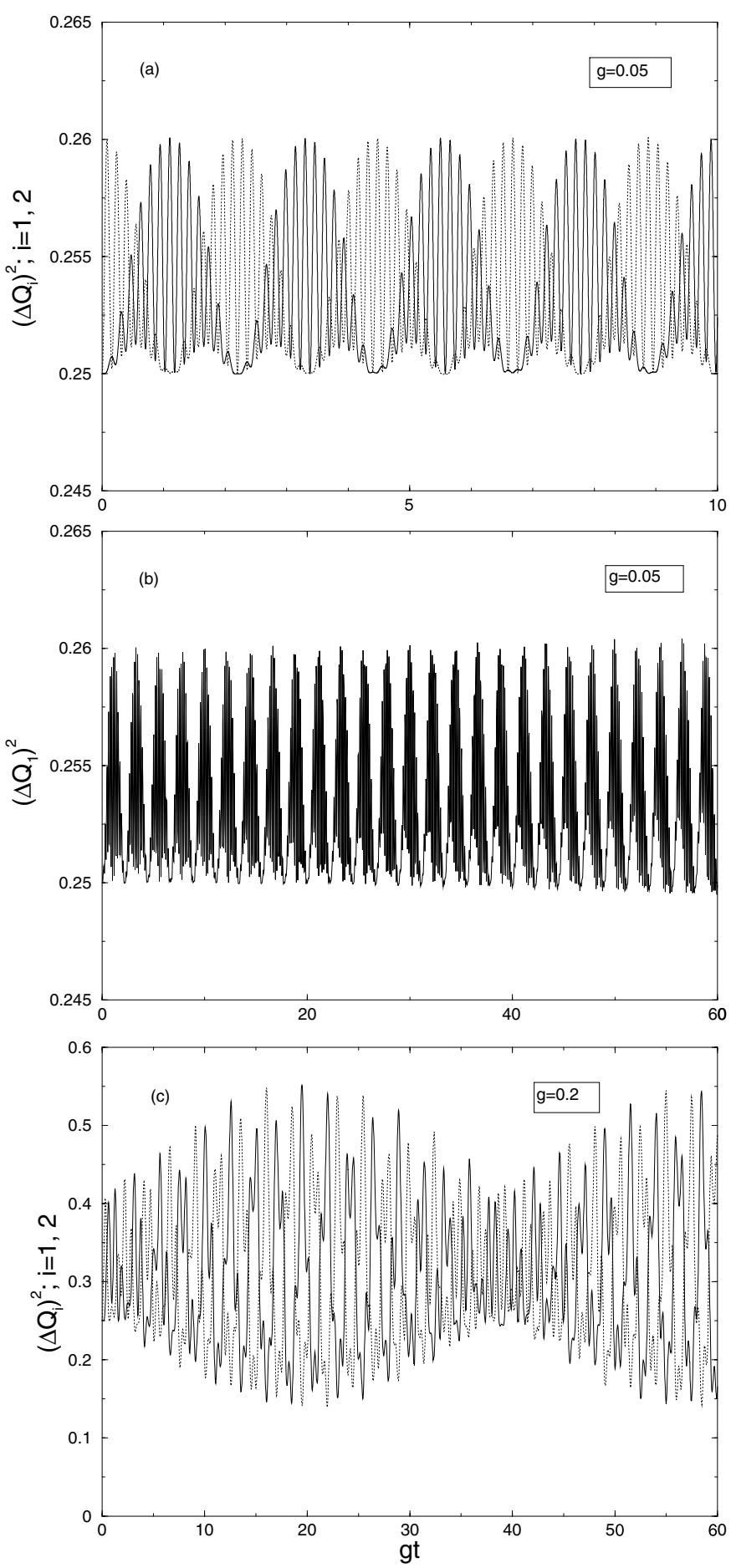

Figure 8. Time evolution of the squeezing variances. Squeezing occurs when $\left(\Delta Q_{1}\right)^{2}$ or $\left(\Delta Q_{2}\right)^{2}<\frac{1}{4}$. (a) $g=0.05$, SUB-30, short time, $(b) g=0.05$, SUB-30, longer time, and $(c)$ $g=0.2$, SUB-14. The Hamiltonian is resonant $\left(\omega=\omega_{0}=1\right)$. 
Table 3. Comparison of NCCM SUB- $N$ results with results obtained from CI diagonalization including the same number of basis states. The table lists the atomic inversion of the resonant Rabi Hamiltonian $\left(\omega=\omega_{0}=1\right)$ for two different couplings. The times, given by $g t$, where the inversion was evaluated, were chosen so that the inversion was near a local maximum.

\begin{tabular}{rrrrrr}
\hline & \multicolumn{2}{c}{$g=0.05 ; g t=1.25$} & & \multicolumn{2}{c}{$g=0.20 ; g t=1.26$} \\
\cline { 2 - 3 }$N$ & CI & NCCM & & CI & NCCM \\
\hline 2 & -0.999999 & -0.981944 & -0.936100 & -0.745812 \\
4 & -0.981757 & -0.981759 & -0.696200 & -0.696457 \\
6 & -0.981759 & -0.981759 & -0.692675 & -0.694392 \\
8 & -0.981759 & -0.981759 & -0.693094 & -0.694211 \\
10 & -0.981759 & -0.981759 & -0.693087 & -0.692888 \\
12 & -0.981759 & -0.981759 & -0.693087 & -0.692909 \\
14 & -0.981759 & -0.981759 & -0.693087 & -0.692926 \\
16 & -0.981759 & -0.981759 & -0.693087 & -0.693384 \\
\hline
\end{tabular}

In assessing the performance of the NCCM in describing this system, it is useful to compare results with those obtained by the CI method using the same basis. The CI method is equivalent to diagonalization in a truncated set of basis states. If we use the same number of states in both CI and NCCM calculations, the two procedures are of approximately the same computational complexity. In table 3 we compare atomic inversions of the Rabi system calculated by both methods for two couplings. We have chosen times for this comparison where the atomic inversion is near a maximum, and thus the state system at these times is as far from the reference state as possible. It should be noted that both sets of $N=16$ results from the CI method may be treated as exact to the precision of table 3 , as they are converged with results for much larger $N$. These results demonstrate several things about the performance of the NCCM in this system. In the region where the NCCM spectrum contains no complex energies, the NCCM describes the system better than the equivalent CI diagonalization, especially for low $(N=2,4)$ truncation levels. This is due to the superior counting of independent excitations in the NCCM [30]. Conversely, the $g=0.2$ results reflect the fact that the presence of the complex energies prevents the NCCM from converging, limiting the accuracy of the NCCM for higher couplings.

The initial aim of applying the NCCM to the Rabi Hamiltonian was to produce an accurate microscopic description of the time evolution of the system across the whole coupling range. This has however not been completely realized due to the incursion of complex energies in the NCCM spectrum. Similar problems occur in the ground-state description. The underlying reasons for this failure of the NCCM are not yet entirely clear to us, and will merit further study. However, it does seem likely that the existence of the Juddian points, at which level crossings occur, indicates the presence of a subtle symmetry whose preservation or breaking is not reflected in our simple choice of reference state. It should be noted that although van der Walt [45] has tried a number of different reference states and operator selections, none of these has yet entirely solved this problem.

Future work includes analysis of the Rabi Hamiltonian with the NCCM in the holomorphic representation, and an extension of the above method to evolution from a coherent state.

\section{Acknowledgments}

CE acknowledges the financial support of a research studentship from the Engineering and Physical Sciences Research Council (EPSRC) of Great Britain. 


\section{References}

[1] Allen L and Eberly J H 1975 Optical Resonance and Two-Level Atoms (New York: Wiley)

[2] Rabi I I 1937 Phys. Rev. 51652

[3] Marshall J T and Pell J L 1981 Phys. Rev. D 24394

[4] Reik H G, Lais P, Stützle M E and Doucha M 1987 J. Phys. A: Math. Gen. 206327

[5] Jaynes E T and Cummings F W 1963 Proc. IEEE 5189

[6] Graham R and Höhnerbach M 1984 Phys. Lett. A 10161

[7] Feranchuk I D, Komarov L I and Ulyanenkov A P 1996 J. Phys. A: Math. Gen. 294035

[8] Ford G W and O'Connell R F 1997 Physica A 243377

[9] Kus M 1985 Phys. Rev. Lett. 541343

[10] Lo C F, Liu K L, Ng K M and Yuen P H 1993 Quantum Semiclass. Opt. 10 L63

[11] Judd B R 1977 J. Chem. Phys. 671174 Judd B R 1979 J. Phys. C: Solid State Phys. 121685

[12] Reik H G, Nusser H and Amarante Ribeiro L A 1982 J. Phys. A: Math. Gen. 153491 Kuś M 1985 J. Math. Phys. 262792 Reik H G and Doucha M 1986 Phys. Rev. Lett. 57787

[13] Bargmann V 1964 Commun. Pure Appl. Math. 14187

[14] Bishop R F, Davidson N J, Quick R M and van der Walt D M 1999 Phys. Lett. A 254215

[15] Benivegna G and Messina A 1987 Phys. Rev. A 353313

[16] Shirley J H 1965 Phys. Rev. B 138979

[17] Eberly J H, Narozhny N B and Sanchez-Mondragon J J 1980 Phys. Rev. Lett. 441323 Narozhny N B, Sanchez-Mondragon J J and Eberly J H 1981 Phys. Rev. A 23236 Yoo H-I, Sanchez-Mondragon J J and Eberly J H 1981 J. Phys. A: Math. Gen. 141383

[18] Milonni P W and Singh S 1993 Adv. At. Mol. Opt. Phys. 401195

[19] Zaheer K and Zubairy M S 1988 Phys. Rev. A 371628

[20] Fang M-F and Zhou P 1995 J. Mod. Opt. 421199

[21] Phoenix S J D 1989 J. Mod. Opt. 361163

[22] Swain S 1973 J. Phys. A: Math. Gen. 6192 Swain S 1973 J. Phys. A: Math. Gen. 61919

[23] Coester F 1958 Nucl. Phys. 7421 Coester F and Kümmel H 1960 Nucl. Phys. 17477

[24] Kümmel H, Lührmann K H and Zabolitzky J G 1978 Phys. Rep. C 361

[25] Bishop R F and Lührmann K H 1978 Phys. Rev. B 173757

[26] Arponen J 1983 Ann. Phys., NY 151311

[27] Arponen J, Bishop R F and Pajanne E 1987 Phys. Rev. A 362519 Arponen J, Bishop R F and Pajanne E 1987 Phys. Rev. A 362539 Arponen J, Bishop R F, Pajanne E and Robinson N I 1988 Phys. Rev. A 371065

[28] Bishop R F 1991 Theor. Chim. Acta 8095

[29] Arponen J and Bishop R F 1991 Ann. Phys., NY 207171 Arponen J and Bishop R F 1993 Ann. Phys., NY 227275 Arponen J and Bishop R F 1993 Ann. Phys., NY 227334

[30] Bishop R F 1998 Microscopic Quantum Many-Body Theories and Their Applications (Lecture Notes in Physics vol 510) ed J Navarro and A Polls (Berlin: Springer) p 1

[31] Bishop R F and Lührmann K H 1982 Phys. Rev. B 265523

[32] Emrich K and Zabolitzky J G 1984 Phys. Rev. B 302049

[33] Čižek J 1966 J. Chem. Phys. 454256

[34] Pople J A 1999 Rev. Mod. Phys. 711267

[35] Bartlett R J 2000 Theor. Chem. Accounts 103273

[36] Marshall W 1955 Proc. R. Soc. A 23248

[37] Zeng C, Farnell D J J and Bishop R F 1998 J. Stat. Phys. 90327

[38] Krüger S E, Richter J, Schulenburg J, Farnell D J J and Bishop R F 2000 Phys. Rev. B 6114607

[39] Hoodbhoy P and Negele J W 1978 Phys. Rev. C 182380

[40] Guha S and Mukherjee D 1991 Chem. Phys. Lett. 18684

[41] Monkhorst H J 1977 Int. J. Quantum. Chem. Symp. 11421

[42] Monkhorst H J 1987 Phys. Rev. A 361544

[43] Sree Latha G and Durga Prasad M 1996 J. Chem. Phys. 1052972

[44] Bishop R F, Davidson N J, Quick R M and van der Walt D M 1996 Phys. Rev. A 544657 
[45] van der Walt D M 1999 PhD Thesis University of Pretoria

[46] Sree Latha G and Durga Prasad M 1993 Theor. Chim. Acta 86511

Sree Latha G and Durga Prasad M 1995 Chem. Phys. Lett. 241215

[47] Rempe G, Walther H and Klein N 1987 Phys. Rev. Lett. 58353

[48] Ruihua X, Gong-Ou X and Dunhuan L 1995 J. Mod. Opt. 422119

[49] Carmichael H J and Walls D F 1976 J. Phys. B: At. Mol. Phys. 91199

[50] Meystre P and Zubairy M S 1982 Phys. Lett. A 89390 